\title{
Predictive semiology of psychogenic non-epileptic seizures in an epilepsy monitoring unit
}

\author{
Andrew J. Duncan ${ }^{1}$ (D) $\cdot$ Ivana Peric $^{2} \cdot$ Ray Boston $^{1,3} \cdot$ Udaya Seneviratne $^{1,2}$ (D)
}

Received: 15 August 2021 / Revised: 12 September 2021 / Accepted: 13 September 2021 / Published online: 22 September 2021

c) Springer-Verlag GmbH Germany, part of Springer Nature 2021

\begin{abstract}
Introduction The diagnosis of psychogenic nonepileptic seizures (PNES) is a common clinical dilemma. We sought to assess the diagnostic value of four ictal signs commonly used in differentiating PNES from epileptic seizures (ES).

Methods We retrospectively reviewed consecutive adult video-electroencephalogram (VEM) studies conducted at a single tertiary epilepsy center between May 2009 and August 2016. Each event was assessed by a blinded rater for the presence of four signs: fluctuating course, head shaking, hip thrusting, and back arching. The final diagnosis of PNES or ES was established for each event based on clinical and VEM characteristics. All ES were pooled regardless of focal or generalized onset. We analyzed the odds ratio of each sign in PNES in comparison to ES with adjustment for repeated measures using logistic regression. Additionally, we calculated the sensitivity, specificity, predictive values, and likelihood ratios of each sign to diagnose PNES.

Results A total of 742 events from 140 VEM studies were assessed. Fluctuating course (odds ratio (OR) 37.37, 95\% confidence interval (CI) 13.56-102.96, $\mathrm{P}<0.0001$ ), head shaking (OR 2.95, 95\% CI 1.26-6.79, $\mathrm{P}=0.012$ ), and hip thrusting (OR $4.28,95 \% \mathrm{CI} 1.21-15.18, \mathrm{P}=0.02$ ) were each significantly predictive of PNES. Fluctuating course had the highest sensitivity (76.16\%). Back arching (OR 1.06, 95\% CI 0.35-3.20, P=0.92) was not significantly associated with PNES.

Conclusion Fluctuating course, head shaking, and hip thrusting are semiological features significantly more common in PNES than ES. Fluctuating course is the most reliable sign. Back arching does not appear to differentiate PNES from ES.
\end{abstract}

Keywords PNES $\cdot$ Video-EEG $\cdot$ Epilepsy $\cdot$ Predictive value $\cdot$ Sensitivity $\cdot$ Diagnosis

\section{Introduction}

Psychogenic non-epileptic seizures (PNES) are a common diagnostic challenge. PNES is commoner in females and coexists in $2-15 \%$ of patients with epilepsy [1-4]. Psychiatric disorders and social stressors are frequent comorbidities [3-6]. Patients with PNES are frequently on at least one antiseizure medication (ASM) at the time of their diagnosis [3-5] and may receive inappropriate medical interventions,

Andrew J. Duncan

andrew.duncan@svha.org.au

1 Department of Clinical Neurosciences, St. Vincent's Hospital Melbourne, Melbourne, VIC, Australia

2 Department of Neurology, Monash Medical Centre, Melbourne, VIC, Australia

3 Department of Clinical Studies, New Bolton Center, School of Veterinary Medicine, University of Pennsylvania, Philadelphia, USA which can lead to deleterious health and financial implications for patients and health systems [7]. Mortality is increased relative to the general population [8], underscoring the importance of early and accurate diagnosis.

Video-electroencephalography monitoring (VEM) is considered the gold standard test for differentiating PNES from epileptic seizures (ES), but it is not widely available. The recent COVID-19 crisis has further exacerbated the shortage of this technology in some regions [9]. As such, assessment of semiology may be the best readily available diagnostic test available to clinicians in many cases, which may include video footage from handheld electronic devices or witness accounts [10-12]. A diagnosis of PNES is often made by clinicians without VEM based on semiology. In isolation this approach has been shown to be poorly discriminatory $[6$, 13-15], although diagnostic accuracy increases with experience and training of the observer, particularly where the observer is a neurologist or epileptologist $[13,16]$. 
Most patients with PNES exhibit stereotypy in semiology, which can include characteristic and reproducible artefactual EEG findings [17-21]. The plethora of signs described in PNES includes fluctuating course, ictal eye closure, crying, duration exceeding two minutes, non-stertorous postictal breathing pattern, lack of amnesia or postictal state, and several motor signs [11, 22-28]. Patients with PNES have more variable seizure durations in comparison to ES [21]. High specificities and lower sensitivities have been reported for many of these signs, although reported values are widely variable, possibly reflecting small cohort sizes. Ictal eye closure, prolonged seizure duration and non-stertorous postictal breathing each have relatively high sensitivity and specificity for PNES [6, 22, 24, 25].

We conducted a retrospective analysis of sequential studies in a single-tertiary epilepsy center to assess four motor signs commonly used in distinguishing PNES from ES. These were head shaking, a fluctuating seizure course, hip thrusting, and back arching. The selected signs have been discussed previously in the literature [11, 23, 26, 29] and are readily observed by bystanders, emergency care providers and in videos, even with suboptimal resolution. We sought to evaluate the utility of these signs in differentiating PNES from ES in a larger cohort. We hypothesized these four signs have high specificity for diagnosing PNES.

\section{Methods}

\section{Setting and participants}

We reviewed all consecutive VEM records at a single center (Monash Medical Centre, Australia) from May 2009 to August 2016. Patients were frequently monitored while sleep-deprived and with reduced or withheld ASM. Patients underwent continuous inpatient VEM for 2-8 days. No additional seizure provocation methods such as placebo injections or photic stimulation were used. Adult ( $>18$ years of age) records that captured seizures were included in the analysis. Patients and events were excluded where incomplete demographic or clinical data were available, if no events occurred during the period of monitoring, where both ES and PNES were captured in the same recording, or where the video recording of an event was of poor quality. All consecutive events were analyzed regardless of the presence or absence of motor semiology, to maximize real-world applicability and to limit selection bias.

This study was approved by the Human Research Ethics Committee of Monash Health, Victoria, Australia.

\section{Data acquisition}

The Compumedics digital EEG system (Compumedics Ltd., Melbourne, Australia) was used for EEG acquisition and review, utilizing the international 10-20 scalp electrode schema. The VEM was reviewed in a multidisciplinary meeting including two epileptologists with access to clinical and imaging data. At this meeting, the final diagnosis of PNES or ES was made. All captured events from each VEM admission were analyzed. For the purpose of the current study, each video was reviewed for the presence or absence of the four signs of interest by two investigators (AD, IP) independently, who were blinded to the final diagnosis.

The signs of interest for the present study were defined as follows: (1) fluctuating course (waxing and waning in amplitude, or non-continuity of motor manifestations); (2) head shaking (side-to-side 'no-no' motion); (3) hip thrusting (alternating anteroposterior pelvic motion); and (4) back arching (obvious and sustained concave posturing with elevation from the bed surface).

Clinical data were collated, including ES subtype based on the ILAE operational classification [30], and the PNES subtype, according to a published schema [17].

\section{Data analysis}

Logistic regression analysis was performed using the Stata statistical software package Version 16.1 (StataCorp LLC, TX, USA). As multiple events were recorded from most subjects, we employed a subject-based clustered robust variance estimation option to offset potential heterogeneities in conjunction with estimation.

$P$ values of $<0.05$ were considered significant. We calculated the specificity, sensitivity, positive predictive value (PPV), negative predictive value (NPV), positive likelihood ratio (PLR) and negative likelihood ratio (NLR) to broadly quantify each sign for diagnosing PNES.

\section{Results}

Of 288 consecutive studies screened from May 2009 to August 2016, 140 VEM studies from 138 patients met our eligibility criteria. There were 755 captured events, of which 13 occurred off-camera and were excluded from the assessment, although other events from the same patients were included. In total, 742 captured events were analyzed (ES 419; PNES, 323). The number of events per VEM recording did not significantly differ between patients with 
Table 1 Epileptic and psychogenic non-epileptic seizure types in the cohort

\begin{tabular}{lc}
\hline Seizure type & Frequency $(\%)$ \\
\hline Epileptic & $419(56.5 \%)$ \\
Focal onset, aware & $18(4.3 \%)-16$ nonmotor onset, 2 motor onset \\
Focal onset with impaired awareness & $270(64.4 \%)$ \\
Focal to bilateral tonic-clonic & $15(3.6 \%)$ \\
Generalized onset, non-motor & $31(7.4 \%)-5$ typical absence, 7 atypical absence, 19 eyelid myoclonia with absences \\
Generalized onset, motor & $80(19.1 \%)-7$ tonic-clonic, 59 tonic, 11 myoclonic, 3 myoclonic-atonic \\
Psychogenic non-epileptic & $323(43.5 \%)$ \\
Rhythmic motor & $88(27.2 \%)$ \\
Hypermotor & $4(1.2 \%)$ \\
Complex motor & $101(31.3 \%)$ \\
Dialeptic & $39(12.1 \%)$ \\
Psychogenic nonepileptic aura & $41(12.7 \%)$ \\
Mixed & $50(15.5 \%)$ \\
\hline
\end{tabular}

PNES and ES $(P=0.459)$. Table 1 provides a breakdown of seizure types captured.

Of 74 patients with ES captured, the mean age was 42.3 years (standard deviation (SD) 17.7, range 18-87.4). The mean number of events per patient was 4.9 (range 1-49). Twenty-six patients (35.1\%) with ES were females.

Of 66 patients with PNES captured, the mean age was 38.9 years (SD 15.3, range 18-84.6). A mean of 5.8 (range $1-29)$ events occurred per patient. Forty-six (69.7\%) were females.

Fluctuating course, head shaking, and hip thrusting were each significantly more likely to be seen in PNES, while the presence of back arching (OR 1.06; 95\% confidence interval (CI) 1.21-15.2; $P=0.92$ ) did not differ significantly between PNES and ES. A fluctuating course was the most reliable sign in differentiating PNES from ES (OR 37.4; 95\% CI 13.6-102.6; $P<0.0001)$, with high sensitivity $(76.2 \%)$ and specificity (92.1\%). Table 2 summarizes the odds ratio, sensitivity, specificity PPV, NPV, PLR and NLR for each of the studied semiologic features.

Probabilities of accurate classification of events with logistic regression were found to be: (1) with a fluctuating course, 85.15\%; (2) with head shaking, 60.65\%; (3) with hip thrusting, 63.07\%; (4) with back arching, $56.47 \%$.

Of 96 patients with back arching, 43 had PNES and 53 ES. The ES with back arching group was predominantly composed of tonic seizures (19), and seizures with focal onset impaired awareness (26), while the remainder comprised focal aware seizure with motor onset (1), and generalized onset tonic-clonic or focal to bilateral tonic-clonic seizures (7). Back arching in the PNES cohort was seen with complex motor (18), rhythmic motor (12), hypermotor (3), and mixed (10) events.

\section{Discussion}

We assessed the discriminatory value of four signs commonly used in differentiating ES and PNES in a large inpatient VEM cohort from a single-tertiary center. A fluctuating (waxing and waning) ictal course, head shaking, and hip thrusting were all significantly more likely to be seen in PNES than ES, with high specificities. Fluctuating course had a relatively high sensitivity, PPV, and NPV, whereas the other studied signs had low sensitivities (Table 2). Back

Table 2 Odds ratio, significance, negative predictive value, and positive predictive value of semiologic signs favoring the diagnosis of PNES

\begin{tabular}{|c|c|c|c|c|c|c|c|c|}
\hline & OR $(95 \% \mathrm{CI})$ & $P$ value & Sensitivity (\%) & Specificity (\%) & PPV (\%) & NPV (\%) & PLR & NLR \\
\hline Fluctuating course & $37.37(13.56-102.96)$ & $<0.0001$ & 76.16 & 92.12 & 88.17 & 83.37 & 9.67 & 0.26 \\
\hline Head shaking & $2.95(1.26-6.79)$ & 0.012 & 19.50 & 92.36 & 66.32 & 59.81 & 2.55 & 0.87 \\
\hline Hip thrusting & $4.28(1.21-15.18)$ & 0.02 & 24.15 & 93.08 & 72.90 & 61.42 & 3.49 & 0.81 \\
\hline Back arching & $1.06(0.35-3.20)$ & 0.92 & 13.31 & 87.35 & 44.79 & 56.66 & 1.05 & 0.99 \\
\hline
\end{tabular}

$O R$ odds ratio, $C I$ confidence interval, $P P V$ positive predictive value, $N P V$ negative predictive value, $P L R$ positive likelihood ratio, $N L R$ negative likelihood ratio 
arching was not significantly associated with PNES, and on logistic regression, the probability of correctly diagnosing PNES using this sign was little better than random chance.

Accurate PNES diagnosis, essential to guide clinical management, may be challenging where VEM does not capture typical events or is unavailable. Smartphone video review, coupled with clinical history and examination, increases the odds of correct diagnosis, providing clinicians with a valuable resource in this setting [31]. Hence, there is a need to identify robust semiological signs for the identification of PNES through video assessment. These signs should be highly specific, to avoid misdiagnosis, while high sensitivity is also required to maximize their applicability. Of the many published PNES signs that may be appreciated in a smartphone video, many are highly specific but have a lower sensitivity [11, 22-28]. Absent postictal deep, heavy breathing (stertor) and ictal eye closure, however, are both highly specific and relatively sensitive findings [22-26, 28]. A fluctuating course is both specific and sensitive for PNES [11, $21,23]$, as confirmed in this study, with comparable value to ictal eye closure and absent postictal stertor. It is a sign relatively easy to appreciate in a video even with suboptimal resolution. We found pelvic thrusting and head shaking to be useful signs for diagnosing PNES, but their relatively low sensitivity in our cohort rendered them less applicable than fluctuating course. Therefore, fluctuating course, ictal eye closure and absent postictal stertorous breathing should be considered reliable PNES features during smartphone video assessment to arrive at a diagnosis.

A fluctuating course was the most reliable of the signs we assessed in diagnosing PNES, with a high OR (37.37), PPV, NPV, and PLR; it was also the most sensitive sign studied (76.16\%) by a wide margin. Logistic regression correctly classified $85.18 \%$ of patients based on this finding alone. Fluctuating or discontinuous ictal motor manifestations have been previously demonstrated to have high specificity for PNES; there is also a relatively high sensitivity in comparison to other semiological features [11,23, 26], particularly where there are two or more pauses during an event (Table 3) [21].

Ictal head shaking is a well-established PNES feature, with a range of reported sensitivities from 25 to $66 \%$ and specificities from 61 to $100 \%$ [11, 25, 26, 28, 32]. In our cohort, head shaking was slightly less sensitive than comparable studies at $19.5 \%$, although specificity was relatively high at $92.4 \%$, in keeping with previous literature.

Hip thrusting has previously been reported to have a sensitivity of $8-44 \%$ for PNES. Specificities reported in or calculated post-hoc from these papers range from 88 to $98 \%$ (Table 3) [11, 25, 28, 32, 33]. Our findings are consistent with published literature in this regard, with a sensitivity of $24.15 \%$ and a specificity of $93.08 \%$.
We found a low sensitivity (13.31\%) and a higher specificity $(87.35 \%)$ for back arching in PNES, similar to previous reports [28, 34], with a low PPV and NPV; the sign was seen with a similar frequency in both PNES and ES and did not approach significance in differentiating PNES from ES $(P=0.92)$. Despite appearing in descriptions of PNES as far back as the nineteenth century, back arching has seldom been critically appraised in the modern scientific literature. A study published in 2011 described sensitivity of $8 \%$ and specificity of $96 \%$. The sign was not internally defined and did not significantly differentiate between PNES and ES $(P=0.894)$ [28]. Another study described back arching in 4 of 53 patients with PNES, but combined ES with organic seizure mimics when comparing to PNES, limiting interpretation [34]. An uncontrolled study reported a sensitivity of $28.6 \%$ [35] and another suggested that back arching is common, without providing quantification [36]. One retrospective analysis suggested a strong PNES association [26], however, the PNES cases were specifically selected to exemplify motor features for a training program, introducing significant selection bias that may have overrepresented back arching, limiting applicability in other settings [26]. Our study represents the largest published cohort assessing this sign. Despite its application in medical teaching as a means of diagnosing PNES, we did not find evidence that this feature reliably differentiates PNES and ES. This may relate to the definition of the sign—arc de cercle posturing, described by Richer and Charcot in 1881 [37], entails arching of the entire body including back, neck and lower limbs; the index patient lifted her entire trunk and pelvis high into the air during events. This degree of arching was not seen in any of our patients, while 'back arching' and 'opisthotonos' are not internally defined by other studies. We found that tonic seizures, particularly, can exhibit back arching. Table 3 summarizes comparable literature for each sign. We suggest that back arching should be defined as clear, sustained arc de cercle posturing in future studies, which may improve the utility and accuracy of this sign in diagnosing PNES.

Our study has a number of strengths. It represents one of the largest published cohorts assessing the described four signs (Table 3), with adjustment for repeated measures from single patients employed. We assessed consecutive VEM patients, rather than selecting exemplar subjects, to limit bias. Assessments were made blinded for each sign.

There are several limitations. Foremost, this is a retrospective single-tertiary center study, thus generalizability is limited. It is not routine practice at our center to refer every patient with suspected PNES for prolonged monitoring where a clear diagnosis has already been reached through other means, for example, a routine EEG record with a typical event captured. As such, the study population may not reflect the full spectrum of PNES presentations. Our consecutive recruitment methodology, 
Table 3 Frequency of described ictal signs in literature

\begin{tabular}{|c|c|c|c|}
\hline $\begin{array}{l}\text { Semiological sign } \\
\text { Publication }\end{array}$ & Sensitivity for PNES & Specificity for PNES & Control group/comment \\
\hline \multicolumn{4}{|l|}{ Fluctuating course } \\
\hline Current study & $76.16 \% *$ & $\mathbf{9 2 . 1 2} \%$ & ES \\
\hline Chen 2008 [11] & $68.75 \% * \dagger$ & $96.30 \% \dagger$ & ES with suspected focal onset \\
\hline Syed 2011 [28] & $42 \%$ & $96 \%$ & ES \\
\hline \multicolumn{4}{|l|}{ Head shaking } \\
\hline Current study & $19.50 \% *$ & $92.36 \%$ & ES \\
\hline Gates 1985 [32] & $36.00 \% *$ & $92.00 \% \dagger$ & BLTCS \\
\hline Azar 2008 [25] & $62.50 \% * \dagger$ & $61.36 \% \dagger$ & $\begin{array}{l}\text { PNES mimicking BLTCS. Non-significant difference compared to } \\
\text { frontal lobe hypermotor ES }\end{array}$ \\
\hline Chen 2008 [11] & $25.00 \% * \dagger$ & $100.00 \% \dagger$ & ES with suspected focal onset \\
\hline Syed $2011[28]$ & $25 \%$ & $87 \%$ & ES \\
\hline \multicolumn{4}{|l|}{ Hip thrusting } \\
\hline Current study & $24.15 \% *$ & $\mathbf{9 3 . 0 8} \%$ & ES \\
\hline Gates 1985 [32] & $44.00 \% *$ & $88.00 \% \dagger$ & Bilateral tonic-clonic seizures versus PNES with motor features \\
\hline Geyer 2000 [33] & $\begin{array}{l}17.00 \% * \dagger \\
78 \% \text { in subset with } \\
\text { hypermotor PNES }\end{array}$ & $88.82 \% \dagger$ & $\begin{array}{l}\text { Present in 0/11 with generalized epilepsy; } 12 / 50 \text { with frontal } \\
\text { epilepsy; } 6 / 100 \text { with TLE }\end{array}$ \\
\hline Azar 2008 [25] & $8.33 \% \dagger$ & $97.73 \% \dagger$ & PNES mimicking BLTCS \\
\hline Chen 2008 [11] & $31.25 \% * \dagger$ & $96.30 \% \dagger$ & ES with suspected focal onset \\
\hline Syed $2011[28]$ & $8 \%$ & $96 \%$ & ES \\
\hline \multicolumn{4}{|l|}{ Back arching } \\
\hline Current study & $13.31 \%$ & $\mathbf{8 7 . 3 5 \%}$ & ES \\
\hline Kotsopoulos 2003 [34] & $7.55 \% *$ & $99.43 \% \dagger$ & $\begin{array}{l}\text { Compared PNES against combined group of ES + organic seizure } \\
\text { mimics }\end{array}$ \\
\hline Syed $2011[28]$ & $8 \%$ & $96 \%$ & ES \\
\hline
\end{tabular}

Results of this study are shown in bold

ES epileptic seizures, PNES psychogenic nonepileptic seizures, TLE temporal lobe epilepsy, BLTCS bilateral tonic-clonic seizures

*Denotes a finding that was reported to reach statistical significance $(P<0.05$ or $95 \%$ confidence interval not including 1$)$ in differentiating PNES and ES

$\dagger$ Denotes a result that was calculated from available information in the referenced article

designed to minimize selection bias, is in keeping with previous similar studies [28]; hence, nonmotor events were assessed alongside events with prominent motor manifestations. Similarly, this study design may, by chance, have led to disproportionate representation of unusual ES types, such as eyelid myoclonia with absence. This study did not differentiate ES subtypes in analysis, limiting applicability to specific situations, for example distinguishing focal ES and PNES. Finally, we did not perform kappa statistics to evaluate interrater agreement.

Our sample size was insufficient to assess whether the simultaneous presence of multiple signs improved specificity. It is common in clinical practice to see multiple PNES signs during a single event, and future research should focus on analyses to appraise the diagnostic value of sign combinations reflecting the real-life scenario.

\section{Conclusion}

In a large cohort of consecutive VEM patients from a single epilepsy center, we found a fluctuating course to be a highly sensitive and specific sign to differentiate PNES from ES. Both hip thrusting and head shaking were also specific, but with a lower sensitivity, limiting applicability in clinical practice. In contrast to these semiologies, we found back arching to be an unreliable sign, concordant with previous studies. Our results provide valuable guidance to differentiate PNES from ES based on semiology, particularly in the settings where VEM is not available.

Author contributions Research project concept AD and US. Literature review and manuscript preparation $\mathrm{AD}$ and US. Data collection $\mathrm{AD}$ 
and IP. Data interpretation US, AD and IP. Statistical analyses and interpretation $\mathrm{AD}$ and $\mathrm{RB}$.

Funding This research did not receive any specific grant from funding agencies in the public, commercial, or not-for-profit sectors.

Availability of data and material Data can be made available upon request, subject to institutional approval.

Code availability Data analysis was performed using STATA software package.

\section{Declarations}

Conflicts of interest US has received travel and speaker honoraria from Eisai Australia and LivaNova Australia. AD, IP and RB report no disclosures.

Ethics approval This project was approved by the Monash Health Human Resource and Ethics Committee.

Consent to participate A waiver of consent was granted, given the retrospective, noninterventional nature of this study.

Consent for publication A waiver of consent was granted.

\section{References}

1. Benbadis SR, Agrawal V, Tatum WOT (2001) How many patients with psychogenic nonepileptic seizures also have epilepsy? Neurology 57:915-917. https://doi.org/10.1212/wnl.57.5.915

2. Martin R, Burneo JG, Prasad A, Powell T, Faught E, Knowlton R, Mendez M, Kuzniecky R (2003) Frequency of epilepsy in patients with psychogenic seizures monitored by video-EEG. Neurology 61:1791-1792. https://doi.org/10.1212/01.wnl.0000098890. 13946.f5

3. Duncan R, Oto M, Martin E, Pelosi A (2006) Late onset psychogenic nonepileptic attacks. Neurology 66:1644-1647. https://doi. org/10.1212/01.wnl.0000223320.94812.7a

4. Lempert T, Schmidt D (1990) Natural history and outcome of psychogenic seizures: a clinical study in 50 patients. J Neurol 237:35-38. https://doi.org/10.1007/BF00319665

5. Lancman ME, Brotherton TA, Asconape JJ, Penry JK (1993) Psychogenic seizures in adults: a longitudinal analysis. Seizure 2:281-286. https://doi.org/10.1016/s1059-1311(05)80141-4

6. Gilmour GS, MacIsaac R, Subotic A, Wiebe S, Josephson CB (2021) Diagnostic accuracy of clinical signs and symptoms for psychogenic nonepileptic attacks versus epileptic seizures: a systematic review and meta-analysis. Epilepsy Behav 121:108030. https://doi.org/10.1016/j.yebeh.2021.108030

7. LaFrance WC Jr, Benbadis SR (2006) Avoiding the costs of unrecognized psychological nonepileptic seizures. Neurology 66:1620 1621. https://doi.org/10.1212/01.wnl.0000224953.94807.be

8. Nightscales R, McCartney L, Auvrez C, Tao G, Barnard S, Malpas CB, Perucca P, McIntosh A, Chen Z, Sivathamboo S, Ignatiadis S, Jones S, Adams S, Cook MJ, Kwan P, Velakoulis D, D'Souza W, Berkovic SF, O'Brien TJ (2020) Mortality in patients with psychogenic nonepileptic seizures. Neurology 95:e643-e652. https:// doi.org/10.1212/WNL.0000000000009855

9. Kuroda N (2020) Decision making on telemedicine for patients with epilepsy during the Coronavirus disease 2019 (COVID-19) crisis. Front Neurol 11:722. https://doi.org/10.3389/fneur.2020. 00722

10. Ramanujam B, Dash D, Tripathi M (2018) Can home videos made on smartphones complement video-EEG in diagnosing psychogenic nonepileptic seizures? Seizure 62:95-98. https://doi.org/10. 1016/j.seizure.2018.10.003

11. Chen DK, Graber KD, Anderson CT, Fisher RS (2008) Sensitivity and specificity of video alone versus electroencephalography alone for the diagnosis of partial seizures. Epilepsy Behav 13:115-118. https://doi.org/10.1016/j.yebeh.2008.02.018

12. Kerr WT, Chau AM, Janio EA, Braesch CT, Le JM, Hori JM, Patel AB, Gallardo NL, Bauirjan J, Allas CH, Karimi AH, Hwang ES, Davis EC, Buchard A, Torres-Barba D, D'Ambrosio S, Al Banna M, Cho AY, Engel J Jr, Cohen MS, Stern JM (2019) Reliability of reported peri-ictal behavior to identify psychogenic nonepileptic seizures. Seizure 67:45-51. https://doi.org/10.1016/j. seizure.2019.02.021

13. Seneviratne U, Rajendran D, Brusco M, Phan TG (2012) How good are we at diagnosing seizures based on semiology? Epilepsia 53:e63-66. https://doi.org/10.1111/j.1528-1167.2011.03382.x

14. Seneviratne U, Ma H, Phan TG (2016) How do doctors in training react to seizures? Epilepsy Behav 54:104-109. https://doi.org/10. 1016/j.yebeh.2015.11.016

15. Erba G, Giussani G, Juersivich A, Magaudda A, Chiesa V, Lagana A, Di Rosa G, Bianchi E, Langfitt J, Beghi E (2016) The semiology of psychogenic nonepileptic seizures revisited: can video alone predict the diagnosis? Preliminary data from a prospective feasibility study. Epilepsia 57:777-785. https://doi.org/10.1111/ epi.13351

16. Wasserman D, Herskovitz M (2017) Epileptic vs psychogenic nonepileptic seizures: a video-based survey. Epilepsy Behav 73:42-45. https://doi.org/10.1016/j.yebeh.2017.04.020

17. Seneviratne U, Reutens D, D'Souza W (2010) Stereotypy of psychogenic nonepileptic seizures: insights from video-EEG monitoring. Epilepsia 51:1159-1168. https://doi.org/10.1111/j.15281167.2010.02560.x

18. Herskovitz M (2017) Stereotypy of psychogenic nonepileptic seizures. Epilepsy Behav 70:140-144. https://doi.org/10.1016/j. yebeh.2017.02.015

19. Beniczky S, Conradsen I, Moldovan M, Jennum P, Fabricius M, Benedek K, Andersen N, Hjalgrim H, Wolf P (2014) Quantitative analysis of surface electromyography during epileptic and nonepileptic convulsive seizures. Epilepsia 55:1128-1134. https:// doi.org/10.1111/epi.12669

20. Vinton A, Carino J, Vogrin S, Macgregor L, Kilpatrick C, Matkovic Z, O'Brien TJ (2004) "Convulsive" nonepileptic seizures have a characteristic pattern of rhythmic artifact distinguishing them from convulsive epileptic seizures. Epilepsia 45:1344-1350. https://doi.org/10.1111/j.0013-9580.2004.04704.x

21. Vogrig A, Hsiang JC, Ng J, Rolnick J, Cheng J, Parvizi J (2019) A systematic study of stereotypy in epileptic seizures versus psychogenic seizure-like events. Epilepsy Behav 90:172-177. https:// doi.org/10.1016/j.yebeh.2018.11.030

22. Seneviratne U, Minato E, Paul E (2017) How reliable is ictal duration to differentiate psychogenic nonepileptic seizures from epileptic seizures? Epilepsy Behav 66:127-131. https://doi.org/ 10.1016/j.yebeh.2016.10.024

23. Avbersek A, Sisodiya S (2010) Does the primary literature provide support for clinical signs used to distinguish psychogenic nonepileptic seizures from epileptic seizures? J Neurol Neurosurg Psychiatry 81:719-725. https://doi.org/10.1136/jnnp.2009.197996

24. Chung SS, Gerber P, Kirlin KA (2006) Ictal eye closure is a reliable indicator for psychogenic nonepileptic seizures. Neurology 66:1730-1731. https://doi.org/10.1212/01.wnl.0000218160. 31537.87 
25. Azar NJ, Tayah TF, Wang L, Song Y, Abou-Khalil BW (2008) Postictal breathing pattern distinguishes epileptic from nonepileptic convulsive seizures. Epilepsia 49:132-137. https://doi.org/ 10.1111/j.1528-1167.2007.01215.x

26. De Paola L, Terra VC, Silvado CE, Teive HA, Palmini A, Valente KD, Olandoski M, LaFrance WC Jr (2016) Improving first responders' psychogenic nonepileptic seizures diagnosis accuracy: development and validation of a 6-item bedside diagnostic tool. Epilepsy Behav 54:40-46. https://doi.org/10.1016/j.yebeh.2015. 10.025

27. Izadyar S, Shah V, James B (2018) Comparison of postictal semiology and behavior in psychogenic nonepileptic and epileptic seizures. Epilepsy Behav 88:123-129. https://doi.org/10.1016/j. yebeh.2018.08.020

28. Syed TU, LaFrance WC Jr, Kahriman ES, Hasan SN, Rajasekaran V, Gulati D, Borad S, Shahid A, Fernandez-Baca G, Garcia N, Pawlowski M, Loddenkemper T, Amina S, Koubeissi MZ (2011) Can semiology predict psychogenic nonepileptic seizures? A prospective study. Ann Neurol 69:997-1004. https://doi.org/10.1002/ ana. 22345

29. Mostacci B, Bisulli F, Alvisi L, Licchetta L, Baruzzi A, Tinuper P (2011) Ictal characteristics of psychogenic nonepileptic seizures: what we have learned from video/EEG recordings - a literature review. Epilepsy Behav 22:144-153. https://doi.org/10.1016/j. yebeh.2011.07.003

30. Fisher RS, Cross JH, French JA, Higurashi N, Hirsch E, Jansen FE, Lagae L, Moshe SL, Peltola J, Roulet Perez E, Scheffer IE, Zuberi SM (2017) Operational classification of seizure types by the International league against epilepsy: position paper of the
ILAE commission for classification and terminology. Epilepsia 58:522-530. https://doi.org/10.1111/epi.13670

31. Tatum WO, Hirsch LJ, Gelfand MA, Acton EK, LaFrance WC Jr, Duckrow RB, Chen DK, Blum AS, Hixson JD, Drazkowski JF, Benbadis SR, Cascino GD, Investigators OS (2020) Assessment of the predictive value of outpatient smartphone videos for diagnosis of epileptic seizures. JAMA Neurol 77:593-600. https://doi.org/ 10.1001/jamaneurol.2019.4785

32. Gates JR, Ramani V, Whalen S, Loewenson R (1985) Ictal characteristics of pseudoseizures. Arch Neurol 42:1183-1187. https:// doi.org/10.1001/archneur.1985.04060110065017

33. Geyer JD, Payne TA, Drury I (2000) The value of pelvic thrusting in the diagnosis of seizures and pseudoseizures. Neurology 54:227-229. https://doi.org/10.1212/wnl.54.1.227

34. Kotsopoulos IA, de Krom MC, Kessels FG, Lodder J, Troost J, Twellaar M, van Merode T, Knottnerus AJ (2003) The diagnosis of epileptic and non-epileptic seizures. Epilepsy Res 57:59-67. https://doi.org/10.1016/j.eplepsyres.2003.10.014

35. Hovorka J, Nezadal T, Herman E, Nemcova I, Bajacek M (2007) Psychogenic non-epileptic seizures, prospective clinical experience: diagnosis, clinical features, risk factors, psychiatric comorbidity, treatment outcome. Epileptic Disord 9(Suppl 1):S52-58. https://doi.org/10.1684/epd.2008.0156

36. Gulick TA, Spinks IP, King DW (1982) Pseudoseizures: ictal phenomena. Neurology 32:24-30. https://doi.org/10.1212/wnl.32.1. 24

37. Richer P (1881) Études Cliniques sur L’Hystéro-Épilepsie ou Grande Hystérie. Delahaye et Lecrosnier, Paris 